\title{
The Interstitial System of the Brain in Health and Disease
}

\author{
Ashok K. Shetty ${ }^{1 *}$, Gabriele Zanirati \\ ${ }^{1}$ Institute for Regenerative Medicine, Department of Molecular and Cellular Medicine, Texas A\&M University \\ College of Medicine, College Station, TX 77843, USA \\ ${ }^{2}$ Brain Institute of Rio Grande do Sul (BraIns), Pontifical Catholic University of Rio Grande do Sul (PUCRS), \\ Porto Alegre, RS, Brazil
}

[Received December 30, 2019; Revised January 3, 2020; Accepted January 3, 2020]

\begin{abstract}
The brain interstitial fluid (ISF) and the cerebrospinal fluid (CSF) cushion and support the brain cells. The ISF occupies the brain interstitial system (ISS), whereas the CSF fills the brain ventricles and the subarachnoid space. The brain ISS is an asymmetrical, tortuous, and exceptionally confined space between neural cells and the brain microvasculature. Recently, with a newly developed in vivo measuring technique, a series of discoveries have been made in the brain ISS and the drainage of ISF. The goal of this review is to confer recent advances in our understanding of the brain ISS, including its structure, function, and the various processes mediating or disrupting ISF drainage in physiological and pathological conditions. The brain ISF in the deep brain regions has recently been demonstrated to drain in a compartmentalized ISS instead of a highly connected system, together with the drainage of ISF into the cerebrospinal fluid (CSF) at the surface of the cerebral cortex and the transportation from CSF into cervical lymph nodes. Besides, accumulation of tau in the brain ISS in conditions such as Alzheimer's disease and its link to the sleep-wake cycle and sleep deprivation, clearance of ISF in a deep sleep via increased CSF flow, novel approaches to remove beta-amyloid from the brain ISS, and obstruction to the ISF drainage in neurological conditions are deliberated. Moreover, the role of ISS in the passage of extracellular vesicles (EVs) released from neural cells and the rapid targeting of therapeutic EVs into neural cells in the entire brain following an intranasal administration, and the promise and limitations of ISS based drug delivery approaches are discussed
\end{abstract}

Key words: beta-amyloid, cerebrospinal fluid, extracellular matrix, extracellular vesicles, glymphatic system, interstitial fluid, phosphorylated tau

\section{Introduction}

The brain is made up of neural cells such as neurons, astrocytes, oligodendrocytes, and microglia, the vasculature comprising arteries, arterioles, capillaries, venules and veins, and the interstitial system (ISS). The brain cells are cushioned and supported by two forms of brain-specific fluids, the brain interstitial fluid (ISF) and the cerebrospinal fluid (CSF) [1,2]. The ISF occupies the brain ISS, whereas the CSF fills the cerebral ventricles and the subarachnoid space $[1,2]$. The brain ISS is a dynamic and complex space connecting the vascular system and neural networks, which is composed of ISF and the extracellular matrix (ECM) (Fig. 1). The ISS is the primary compartment of the brain microenvironment that provides the immediate accommodation space for neural cells, which accounts for $15-20 \%$ of the overall brain volume [1,2]. The space between the adjacent neurons, contiguous glia, or the adjoining neurons and glia is also known as the extracellular space (ECS) [1-3].

*Correspondence should be addressed to: Dr. Ashok K. Shetty, Department of Molecular and Cellular Medicine, Texas A\&M Health Science Center, College of Medicine, College Station, TX 77843, USA. E-mail: akskrs@tamu.edu

Copyright: (c) 2020 Shetty A et al. This is an open-access article distributed under the terms of the Creative Commons Attribution License, which permits unrestricted use, distribution, and reproduction in any medium, provided the original author and source are credited. 


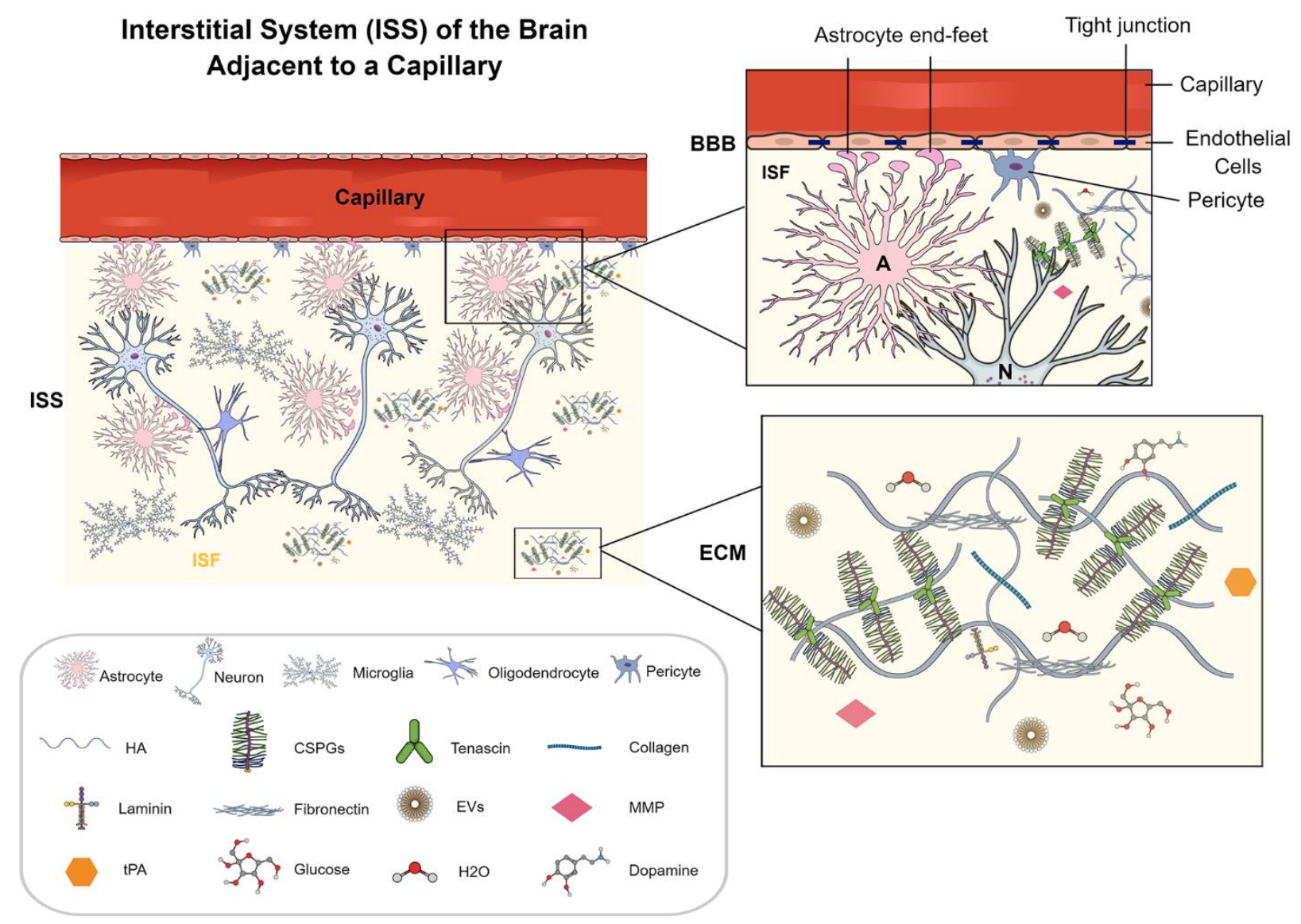

Figure 1. A cartoon illustrating the brain interstitial system (ISS) between neural cells comprising interstitial fluid (ISF) and the extracellular matrix (ECM), adjacent to a brain microvasculature. The magnified view of a portion of the cartoon on the top right shows endothelial cells with tight junctions, astrocyte end-feet, and pericytes at the interface of ISS and the microvasculature. The magnified view on the bottom right shows the ECM with its structure and components. The ECM contains hyaluronic acid, proteoglycans - CSPGs, tenascin, and a small amount of collagen, laminin, and fibronectin. Some components of ISF are represented, including EVs, H2O, glucose, dopamine, matrix metallopeptidase (MMP), and tissue plasminogen activators (tPAs). The illustrated ECM is distributed as the neural interstitial matrix in the space between neural cells. A, astrocyte; BBB, blood-brain barrier; $\mathrm{N}$, neuron.

\section{The geometry of the brain ISS}

The ISS is circumscribed by the plasma membrane of neurons or glia and the wall of blood vessels [4] (Fig. 1). However, the magnitude, locus, and proportions of the brain ISS are not static. Significant transformations ensue in ISS during the development of the brain due to events such as neurogenesis, neuronal migration and differentiation, expansion of dendritic tree, synaptogenesis, gliogenesis, synaptic stripping by microglia, and myelination [1,5-10]. The geometry of the brain ISS also undergoes modification in aging due to the loss of synapses, neurons, dendritic regression, changes in morphology and numbers of glia, senoinflammation, and deposition of amyloid plaques [11-15]. In conditions such as epilepsy, the synchronized firing of a population of neurons may considerably change the geometry of ISS as well as the ISF drainage [16]. Such changes may also contribute to the maintenance of epilepsy because an accumulation of macromolecules within the brain parenchyma could alter the extracellular ionic equilibrium, and impact neuronal excitability [17].

\section{Composition of ISS}

The constituents of the ISS comprise ISF, ECM, and diffusely distributed interstitial matrix (ISM) [1] (Fig. 1). The brain ISF, a water solvent containing ions, and gaseous and organic molecules such as proteins, peptides, enzymes, dopamine, and extracellular vesicles (EVs) secreted by cells, and the moving chains of glycoproteins attached to the ECM (Fig. 1). The ISF provides a direct 
medium for the supply of nutrients, removal of waste, and intercellular communication by encasing and incessantly soaking the neural cells [1, 18-20]. The source of ISF is still being investigated. Some studies have implied that ISF originates from the cerebrospinal fluid (CSF), cell metabolism, and the vascular system [21-24]. The exchange of substances between the ISS and the neural cells happens continuously [25]. A higher osmolarity in neural cells guides water molecules from the brain ISS into neural cells via aquaporin 4 (AQP4) and $\mathrm{Na}^{+} / \mathrm{K}^{+}$ATPase enzymes in the cell membrane [26, 27].

The ECM, an abundantly hydrated net-like assembly produced by cells and disintegrated by enzymes, comprises collagen, elastin, glycosaminoglycans, proteoglycans, and glycoproteins such as tenascin, reelin, laminin, and fibronectin [1, 28-30] (Fig. 1). Besides, the ECM contains perineuronal nets (PNNs) and pericapillary matrix (PCM). PNNs, condensed mesh-like structures comprising chondroitin sulfate proteoglycans, hyaluronic acid, linking proteins, and tenascin-R, mostly encircle GABA-ergic neurons in various brain regions [31, 32]. The elements of PNNs are dynamic, which play an essential role in synaptic plasticity and protecting recent and remote contextual memory through regulation of GABA release in inhibitory interneurons [32]. The constitution of PNNs gets altered in situations such as fear, reward, and stress [33, 34]. The integrity of the PNN is also noticeably disturbed during trauma, epilepsy, tumor, and schizophrenia [35-42]. Also, digestion or removal of PNNs can interfere with the functional recovery after CNS lesions [43]. The PCM is a basement membrane comprising a sheet-like layer made up of collagen IV, laminins, fibronectin, dystroglycan, and perlecan, which is contiguous with the pia mater of the brain [44]. The ECM consists of proteoglycans, hyaluronic acid, and a small amount of collagens, laminins, and fibronectin.

\section{Relationship between the ISF, CSF, and the blood- brain barrier}

The cerebrospinal fluid (CSF) resides in the subarachnoid space covering the entire surface of the brain and the brain ventricles. The CSF serves as a reservoir for the ISF, and an extensive communiqué between the ISF and CSF helps in the removal of waste products from the brain ISF [1, 45] (Fig. 2). Such an exchange between CSF and ISF is vital for the preservation of homeostasis in the neural microenvironment [46]. As per one estimate, $\sim 20 \%$ of CSF in the human brain originates from the brain ISF [1, 47, 48]. A study by Iliff colleagues showed that subarachnoid CSF passes into the brain rapidly, alongside the perivascular spaces adjoining the penetrating arteries and reaches the level of capillaries. Then, aquaporin-4 channels positioned on the perivascular end-feet of astrocytes enable the convective flow of CSF into the ISS. The CSF mixes with the ISF and leaves the brain ISS via perivascular spaces along veins [23] (Fig. 2). A similar process likely also aids the quick entry of nanosized EVs into the brain when administered intranasally. Recent studies have shown that intranasally administered stem cell-derived EVs permeate the entire forebrain and incorporate into different neural cells within 6 hours [49, 50]. Such rapid targeting by EVs is likely due to their quick entry into the subarachnoid space through perineurial spaces around olfactory nerves passing through the cribriform plate and subsequent transportation through CSF flow into the ISF. However, real-time monitoring of EV kinetics in vivo using single-photon emission computed tomography (SPECT)/positron emission tomography (SPECT/PET), and magnetic resonance imaging (MRI) will be needed to validate the above possibility.

Some exchange of substances also occurs between the ISF and the cytoplasm of neural cells as the plasma membrane of neurons and glia is selectively permeable to specific ions and molecules [51-55] (Fig. 2). Moreover, the interchange of constituents occurs between the plasma and the ISF through capillaries [56]. Typically, neurons and capillaries are separated by $10-20 \mu \mathrm{m}$ space, which facilitates a highly efficient diffusion-based substance exchange between capillaries and neurons by a highly selective blood-brain barrier (BBB) [57]. The BBB is composed of capillary endothelial cells, pericytes, and astrocyte end-feet with tight junctions linking the endothelial cells [58, 59] (Fig. 2). The BBB freely allows the passage of water and small hydrophobic molecules such as oxygen, carbon dioxide, and hormones. Besides, the BBB permits the passage of lipid-soluble molecules through simple diffusion, and glucose and amino acids via active transport $[60,61]$.

\section{The function of the brain ISS}

The ISS of the brain is dynamic, which provides a route for cross-talk between elements of the vascular system and neural cells. The role of ISS includes the facilitation of communication between neural cells, processing and integration of information, and coordinated response to changes in the brain environment [1, 62-66]. Furthermore, the peptides and proteins created in the brain ISS are related to the proteolytic processes involved in cell surface remodeling, protein shedding, and the synthesis of regulatory peptides [67]. It has been suggested that the long-distance passage of such active proteins by ISF-CSF exchange is essential for the onset and maintenance of specific behavioral or motivational states such as fear, appetite, mood, and circadian rhythms [68]. Moreover, 
the enzymes in the brain ISF degrade and remodel the various ECM proteins secreted by neurons, glia and endothelial cells, which include matrix metallopeptidases (MMPs), and tissue plasminogen activators (tPAs) [69]. Some of these proteins have essential functions. For example, MMPs play a role in learning and memory [70], synaptic plasticity, and repair after brain disorders [71]. tPAs, on the other hand, are involved in neuronal plasticity, ECM degradation, microglial activation, and accumulation of amyloid plaques [72]. Besides, a recent investigation has shown that the brain ISF accumulation of tau, a protein involved in neurodegeneration in Alzheimer's disease (AD) and tauopathies, is regulated by the sleep-wake cycle [73]. The study also showed that sleep deprivation induces a two-fold increase in the ISF tau, and facilitates tau spreading from the hippocampus into the entorhinal cortex and the locus coeruleus, a region involved in the maintenance of wakefulness [73]. The above observations are consistent with the studies showing the accumulation of beta-amyloid in the healthy brain after a single night of sleep deprivation [74]. Another relevant recent finding is that deep sleep (i.e., during non-rapid eye movement sleep) is associated not only with low-frequency oscillations in neuronal activity and blood oxygenation but also macroscopic changes in CSF flow through the ISS [75]. Such changes in CSF flow can substantially augment ISF volume and removal of metabolic waste products because pulsatile fluid dynamics potentially enhances mixing, diffusion, and clearance of ISF. Because of the lack of lymphatic structures within the brain, the transport and metabolism of substances occur mostly within ECS, which plays a crucial role in many physiological processes such as sleep, memory, and sensory perception. Also, ECS is related to the occurrence and development of major diseases such as $\mathrm{AD}$, Parkinson's disease, and brain tumors. Therefore, it is of immense clinical significance to study the transport of substances within the ECS of the brain.

The ISS also facilitates the passage of neural cell derived EVs into local and distant sites in the brain and to the bloodstream. EVs are broadly classified as microvesicles (MVs) having a size of 100-1000 nm and exosomes (EXs) ranging in size from 30-150 nm. Microvesicles directly bud-off from the plasma membrane of cells [76, 77] whereas, EXs start in endosomes as intraluminal vesicles, leading to the development of multi-vesicular bodies (MVBs) and then secretion of EXs into the ECS via fusion of MVBs with the plasma membrane [78]. EVs are delineated by a phospholipid bilayer and carry nucleic acids and proteins from parental cells [77]. EVs move through the brain ISS and the CSF, contribute to intercellular communication and impact activities such as neurodegeneration, synaptic plasticity, and behavior [79]. Furthermore, studies have shown that information on specific biomarkers in brain disorders can be gleaned by investigating the composition of CNS-derived EVs from the CSF or the blood. It is also possible to analyze the composition of EVs secreted from specific brain cells (e.g., EVs derived from neurons vis-àvis astrocytes). Indeed, evaluation of the composition of EVs released by neurons and glia into the ISF, CSF, or blood has provided diagnostic and prognostic insights on brain function in many neurological disorders [80-85].

\section{Drainage of ISF from the brain ISS}

The biophysical and biochemical balance between CSF and ISF is crucial for the metabolism of brain cells and drugs, and hence, continuous exchanges between CSF and ISF are critical [1]. Three potential conduits have been suggested for the drainage of ISF from the ISS. One path is via the wall of ventricles through ependymal cells, which allows the passage of macromolecules [10, 46, 86] (Fig. 2). Ependymal cells, covered in a layer of cilia, line the ventricles, and play an essential role in the regulation of CSF production. The second passageway is at the surface of the brain and spinal cord via pia-glial membranes [10, 23, 46, 87] (Fig. 2). A direct exchange between the ISF and CSF at these two conduits have been confirmed through the newly developed tracer-based MRI technique and traditional optical imaging techniques [88]. The other pathway is the blood vessel wall, where ISF flows in the basement membrane directly opposite to the blood flow and reaches the extracranial lymph nodes through the ISF clearance pathway or paravascular system [21, 89-91] (Fig. 2). Studies have revealed lymphatic vessels in the CNS carrying lymph into deep cervical lymph nodes [92]. A widespread web of meningeal lymphatic vessels in the base of the skull makes up the ISS, which facilitates the drainage of metabolic waste products from the ISS of the CNS into the bloodstream. The ISF pathway is mostly active during sleep when the clearance of harmful metabolites such as beta-amyloid increases two-fold relative to the waking state [74]. By using a newly developed in vivo tracer-based MRI technique, Han and his group demonstrated the drainage of ISF from deep brain regions into the surface of the cerebral cortex [10, 46, 93]. In addition, they showed that the traced ISF from the caudate nucleus drained into the ipsilateral cerebral cortex along the myelin fiber tracts and then into the subarachnoid space [10]. A study using an in vivo two-photon imaging also verified the ISF-CSF exchange between the ISS of the cerebral cortex and the CSF in the subarachnoid space [23]. 

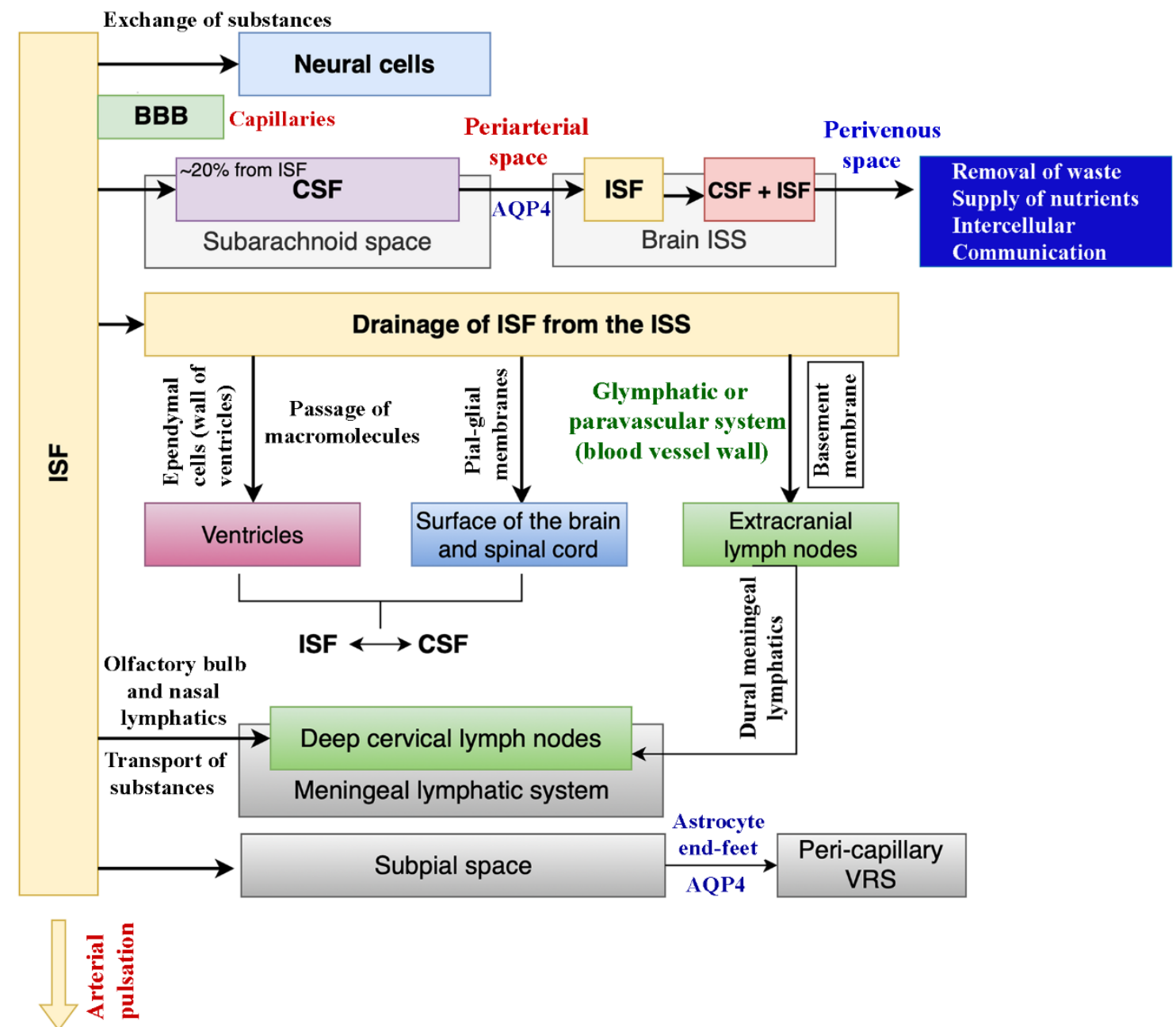

\section{Bulk flow}

Figure 2. A schematic showing the various communications of the interstitial fluid (ISF), and the mechanisms by which ISF is drained into the lymphatic system. ISF interacts with neurons through the capillaries, and the substance exchange between capillaries and neurons is facilitated by the blood-brain barrier (BBB). The cerebrospinal fluid (CSF) resides in the subarachnoid space, and $\sim 20 \%$ of the CSF in the brain comes from the ISF. The CSF influx into the brain happens through the periarterial spaces, the convective flow into the ISS occurs through the water channel aquaporin-4 (AQP4, located on astrocyte end-feet) where CSF mixes with the ISF, and then the efflux of ISF occurs through perivenous spaces. The drainage of ISF occurs through three suggested conduits. These include passage through ependymal cells into ventricles, pia-glial membranes into the surface of the brain and spinal cord, and the glymphatic system into extracranial lymph nodes. The ISF is then transported via dural meningeal lymphatics into the deep cervical lymph nodes. Also, the transportation of substances from the ISF into the deep cervical lymph nodes can occur via the olfactory bulb and nasal lymphatics. Furthermore, the end-feet of astrocytes are also involved in the transportation of ISF from the subpial space into the peri-capillary Virchow-Robin space (VRS) through AQP4 channels. The arterial pulsation promotes the ISF bulk flow.

Kiviniemi and associates, using ultra-fast MRI techniques have demonstrated that three discrete pulsation processes in the human glymphatic system facilitate the exchange between ISF and CSF [94]. The swiftest process is the cardiovascular pulsation commencing from basal peri-arterial spaces in the region of the circle of Willis and outspreading centrifugally towards the cerebral cortex. The other two processes include the respiration related pulses dominating in peri-venous spaces in a centripetal fashion and the slow vasomotor wave fluctuations having distinct spatiotemporal patterns [94]. Nakada and Kwee also suggest the involvement of an astrocyte-based system in ISF drainage through water influx into the pericapillary Virchow-Robin space (VRS). They suggest that the astrocyte system moves the brain ISF from the subpial space into the peri-capillary VRS by the water channel 
aquaporin-4. They also suggest that ISF flow in the arterial VRS is essential for the clearance of beta-amyloid, whereas the ISF flow in the peri-venous VRS creates a bulk flow of ISF and makes up the necessary volume of CSF together with the CSF produced by the choroid plexus [95]. Another recent study by Teng et al. showed differential effects between the thalamus and the caudate nucleus following aquaporin-4 knockout (KO) in rats. In the thalamus, $\mathrm{KO}$ of aquaporin- 4 prolonged the half-life of ISF with a decrease in the clearance coefficient but with no changes in the tortuosity of the ISS [96]. In contrast, in the caudate nucleus, $\mathrm{KO}$ of aquaporin- 4 increased the volume fraction of the ISS and the diffusion coefficient with decreased tortuosity but no changes in brain ISF drainage. The authors attribute this discrepancy in ISF drainage between the two brain regions to the variable distribution of astrocytes and the extent of aquaporin- 4 decline [96].

In conditions such as hydrocephalus, increased ventricular pressure causes high ependymal permeability and enhances the flow from CSF to ISF, which leads to interstitial edema of white matter [97]. However, hydrocephalus does not induce edema in the gray matter of the cerebral cortex because of the more efficient drainage from ISF to CSF via pia-glia membranes [2]. A study, using tracer-based MRI, showed that ISF drainage slows down with increased tortuosity of the ISS in the rotenone-induced rat model of Parkinson's disease. Administration of antiparkinsonian drug madopar partially prevented these changes in ISS and ISF [98]. Another study investigated the effect of olfactory stimulation with eugenol on ISF drainage in the hippocampus and its link with aquaporin 4 [99]. Eugenol significantly increased the activity of hippocampal neurons but reduced the clearance and diffusion rates of Gd-DTPA and A-594 in the hippocampus, implying an interference with the ISF drainage. Eugenol inhalation also slowed down the rate of Gd-DTPA in CSF entering the hippocampus and its clearance. Remarkably, the KO of aquaporin 4 aggravated these processes [99]. Thus, olfactory stimulation can alter the ISF drainage.

\section{Status of ISS in neurological disorders}

The pathological changes in conditions such as glioma or stroke can alter multiple aspects of the ISS, which may include changes in geometry, ECM and its components, the constituents of the ISF, and the transport of substances in the ISS $[1,100,101]$. In glioma, the uncontrolled growth of tumor cells disrupts the standard architecture of brain tissue. Such growth is also accompanied by the degradation of ECM and the active migration of tumor cells [102]. Studies have reported increased volume and/or tortuosity of the ISS in different types of glioma
$[103,104]$. A recent study using a tracer-based MRI has also shown that implantation of glioma into the thalamus slows-down ISF drainage and alters the direction of ISF drainage [105].

In ischemic stroke, studies have shown decreased ISS volume as a result of cytotoxic edema [106], and increased ISS tortuosity due to a reduction in diffusion caused by dead-space in the microdomains of ISS [107109]. Also, aquaporin 4 knockout aggravates early brain injury following subarachnoid hemorrhage through impairment of the glymphatic system in the rat brain [110]. In AD, beta-amyloid deposits accumulate in the brain ISS [111-113], which increases the volume and tortuosity of ISS. It is believed that blockage of brain ISF drainage accelerates the abnormal deposition of betaamyloid in $\mathrm{AD}$, which contributes to both cerebral amyloid angiopathy and neurodegeneration [23, 114, 115]. Changes in the ISS have also been reported in other diseases, such as Parkinson's disease [116], multiple sclerosis [117, 118], and epilepsy [17, 119]. Moreover, another study in a mouse model of AD by Yue and associates suggested that the destruction of beta-amyloid deposits in the ECS using a light-emitting diode with red light facilitated not only the recovery of ISF drainage but also rescued cognitive function [15]. This study suggested a new avenue for treating $\mathrm{AD}$ by targeting beta-amyloid in the ISS. Another study suggested that alcohol intake at low-dose levels promotes the clearance of metabolic waste products, including neurodegenerative diseaserelated proteins through the ISS [120].

\section{ISS as the route of drug delivery - Available methods and limitations}

The failure rate of CNS drugs in both preclinical and clinical studies is much higher than non-CNS drugs [1, $121,122]$. In fact, in conditions such as neurodegenerative diseases, the clinical failure rate for disease-modifying treatments has been $100 \%$ [123]. Also, studies have consistently shown limited penetration into the brain tissue when drugs are delivered intravenously. Therefore, it is plausible that the failure of many potentially effective therapeutics for brain disorders is the result of the inability to effectively deliver and sustain adequate drug concentrations within the brain [124]. From this perspective, ISS based drug delivery approaches have considerable interest. One such promising method is called the convection-enhanced delivery (CED), which involves the continuous injection of the therapeutic agent under positive pressure via a catheter implanted into the brain [125-127]. CED is superior to conventional drug delivery approaches as it facilitates the BBB bypass and enhanced ISF drainage with minimal systemic toxicity and better efficacy. CED has been employed for 
delivering chemotherapeutic agents in several tumor clinical trials and glial cell line-derived neurotrophic factor in Parkinson's disease [128].

Nonetheless, further refinements are necessary for CED to be translated successfully to the clinic. Notably, a device that avoids the backflow or reflux of injection and mechanical damage to the brain tissue is needed [129131]. Furthermore, appropriate in vivo monitoring methods are required to measure the concentration and distribution of the injected drug [132-134]. Simple diffusion delivery (SDD) is another local brain drug delivery method that utilizes the concentration gradients to drive pharmaceutical agents to target zones via the brain ISS [135]. Since the brain ISS is divided into different segments based on brain ISF drainage [88, 92, $118,135]$, SDD has promise for delivering drugs to specific regions of the brain. However, before targeting drugs to brain regions through an SDD method, the tracerbased MRI technique may need to be used to ensure the brain ISF drainage in the area of the brain where the drug needs to be targeted. One of the significant advantages of SDD is that it can facilitate a full immersion and contact of the drug with target cells, which may allow effective treatment with much smaller doses of the drug $[1,136]$.

\section{Conclusions}

Many exciting advances occurred in the past decade regarding our understanding of brain ISS. The new developments in the field include identification of differential ISF drainage in distinct functional segments of the brain, and several routes and mechanisms underlying the drainage of ISF into ventricles and the subarachnoid space. Interestingly, tau accumulation in the brain ISS and sleep deprivation augmenting that process have been identified as one of the factors that facilitate the spread of tau in AD. Additional studies have also shown the promise of the removal of beta-amyloid from the brain ISF for improving cognitive function in $\mathrm{AD}$. On the other hand, ISS-based drug delivery, though promising, needs further development and refinement for a successful clinical application of this approach.

\section{Acknowledgments}

Authors are supported by grants from the National Institute of Neurological Disorders and Stroke (1R01NS106907-01 to A.K.S.) and the Department of Defense (W81XWH-14-1-0572 and W81XWH-16-10480 to A.K.S.). Gabriele Zanirati is supported by a postdoctoral fellowship from Coordenação de Aperfeiçoamento de Pessoal de Nível Superior (CAPES), Government of Brazil.

\section{References}

[1] Lei Y, Han H, Yuan F, Javeed A, Zhao Y (2017). The brain interstitial system: Anatomy, modeling, in vivo measurement, and applications. Prog Neurobiol, 157:230-246.

[2] Chatterjee K, Esparza CM, Munson JM (2019). Methods to measure, model and manipulate fluid flow in brain. J Neurosci Methods: 108541

[3] Pajevic S (2019). Exploring the Dynamics of Brain Extracellular Space. Biophys J, 117):1781-1782.

[4] Nicholson C, Kamali-Zare P, Tao L (2011). Brain Extracellular Space as a Diffusion Barrier. Comput Vis Sci, 14:309-325.

[5] Bielas S, Higginbotham H, Koizumi H, Tanaka T, Gleeson JG (2004). Cortical neuronal migration mutants suggest separate but intersecting pathways. Annu Rev Cell Dev Biol, 20:593-618.

[6] Faissner A, Pyka M, Geissler M, Sobik T, Frischknecht $\mathrm{R}$, Gundelfinger ED, et al. (2010). Contributions of astrocytes to synapse formation and maturation Potential functions of the perisynaptic extracellular matrix. Brain Res Rev, 63:26-38.

[7] Florio M, Huttner WB (2014). Neural progenitors, neurogenesis and the evolution of the neocortex. Development, 141:2182-2194.

[8] Kettenmann H, Kirchhoff F, Verkhratsky A (2013). Microglia: new roles for the synaptic stripper. Neuron, 77:10-18.

[9] Yang S, Wang Y, Li K, Tang X, Zhang K, Shi C, et al. (2016). Extracellular space diffusion analysis in the infant and adult rat striatum using magnetic resonance imaging. Int J Dev Neurosci, 53:1-7.

[10] Wang A, Wang R, Cui D, Huang X, Yuan L, Liu H, et al. (2019). The Drainage of Interstitial Fluid in the Deep Brain is Controlled by the Integrity of Myelination. Aging Dis, 10:937-948.

[11] Dumitriu D, Hao J, Hara Y, Kaufmann J, Janssen WG, Lou $\mathrm{W}$, et al. (2010). Selective changes in thin spine density and morphology in monkey prefrontal cortex correlate with aging-related cognitive impairment. J Neurosci, 30:7507-7515.

[12] Mattson MP, Arumugam TV (2018). Hallmarks of Brain Aging: Adaptive and Pathological Modification by Metabolic States. Cell Metab, 27:1176-1199.

[13] Shetty AK, Kodali M, Upadhya R, Madhu LN (2018). Emerging Anti-Aging Strategies - Scientific Basis and Efficacy. Aging Dis, 9:1165-1184.

[14] Shetty AK, Upadhya R, Madhu LN, Kodali M (2019). Novel Insights on Systemic and Brain Aging, Stroke, Amyotrophic Lateral Sclerosis, and Alzheimer's Disease. Aging Dis, 10:470-482.

[15] Yue X, Mei Y, Zhang Y, Tong Z, Cui D, Yang J, et al. (2019). New insight into Alzheimer's disease: Light reverses Abeta-obstructed interstitial fluid flow and ameliorates memory decline in APP/PS1 mice. Alzheimers Dement (N Y), 5:671-684.

[16] Marchi N, Banjara M, Janigro D (2016). Blood-brain barrier, bulk flow, and interstitial clearance in epilepsy. 
J Neurosci Methods, 260:118-124.

[17] Noe FM, Marchi N (2019). Central nervous system lymphatic unit, immunity, and epilepsy: Is there a link? Epilepsia Open, 4:30-39.

[18] Deitmer JW, Rose CR (1996). $\mathrm{pH}$ regulation and proton signalling by glial cells. Prog Neurobiol, 48:73103.

[19] Fuxe K, Dahlstrom AB, Jonsson G, Marcellino D, Guescini M, Dam M, et al. (2010). The discovery of central monoamine neurons gave volume transmission to the wired brain. Prog Neurobiol, 90:82-100.

[20] Howell MD, Gottschall PE (2012). Lectican proteoglycans, their cleaving metalloproteinases, and plasticity in the central nervous system extracellular microenvironment. Neuroscience, 217:6-18.

[21] Abbott NJ (2004). Evidence for bulk flow of brain interstitial fluid: significance for physiology and pathology. Neurochem Int, 45:545-552.

[22] Brinker T, Stopa E, Morrison J, Klinge P (2014). A new look at cerebrospinal fluid circulation. Fluids Barriers CNS, 11:10.

[23] Iliff JJ, Wang M, Liao Y, Plogg BA, Peng W, Gundersen GA, et al. (2012). A paravascular pathway facilitates CSF flow through the brain parenchyma and the clearance of interstitial solutes, including amyloid beta. Sci Transl Med, 4:147ra111.

[24] Magdoom KN, Brown A, Rey J, Mareci TH, King MA, Sarntinoranont M. 2019. MRI of Whole Rat Brain Perivascular Network Reveals Role for Ventricles in Brain Waste Clearance. Sci Re, 9:11480.

[25] Ding F, O'Donnell J, Xu Q, Kang N, Goldman N, Nedergaard M (2016). Changes in the composition of brain interstitial ions control the sleep-wake cycle. Science, 352:550-5.

[26] Tian J, Cai T, Yuan Z, Wang H, Liu L, Haas M, et al. (2006). Binding of Src to $\mathrm{Na}+/ \mathrm{K}+$-ATPase forms a functional signaling complex. Mol Biol Cell, 17:317326.

[27] Yuan Z, Cai T, Tian J, Ivanov AV, Giovannucci DR, Xie Z (2005). Na/K-ATPase tethers phospholipase C and IP3 receptor into a calcium-regulatory complex. Mol Biol Cell, 16:4034-4045.

[28] Crocker SJ, Pagenstecher A, Campbell IL (2004). The TIMPs tango with MMPs and more in the central nervous system. J Neurosci Res, 75:1-11.

[29] Lau LW, Cua R, Keough MB, Haylock-Jacobs S, Yong VW (2013). Pathophysiology of the brain extracellular matrix: a new target for remyelination. Nat Rev Neurosci, 14:722-729.

[30] Michel G, Tonon T, Scornet D, Cock JM, Kloareg B (2010). The cell wall polysaccharide metabolism of the brown alga Ectocarpus siliculosus. Insights into the evolution of extracellular matrix polysaccharides in Eukaryotes. New Phytol, 188:82-97.

[31] Frischknecht R, Heine M, Perrais D, Seidenbecher CI, Choquet D, Gundelfinger ED (2009). Brain extracellular matrix affects AMPA receptor lateral mobility and short-term synaptic plasticity. Nat Neurosci, 12:897-904.

[32] Shi W, Wei X, Wang X, Du S, Liu W, Song J, et al.
(2019). Perineuronal nets protect long-term memory by limiting activity-dependent inhibition from parvalbumin interneurons. Proc Natl Acad Sci U S A. Gogolla N, Caroni P, Luthi A, Herry C (2009). Perineuronal nets protect fear memories from erasure. Science, 325:1258-1261.

[34] Xue YX, Xue LF, Liu JF, He J, Deng JH, Sun SC, et al. (2014). Depletion of perineuronal nets in the amygdala to enhance the erasure of drug memories. $\mathrm{J}$ Neurosci, 34:6647-6658.

[35] Balmer TS, Carels VM, Frisch JL, Nick TA (2009). Modulation of perineuronal nets and parvalbumin with developmental song learning. J Neurosci, 29:1287812885.

[36] Bavelier D, Levi DM, Li RW, Dan Y, Hensch TK (2010). Removing brakes on adult brain plasticity: from molecular to behavioral interventions. J Neurosci, 30:14964-14971.

[37] Gundelfinger ED, Frischknecht R, Choquet D, Heine M (2010). Converting juvenile into adult plasticity: a role for the brain's extracellular matrix. Eur J Neurosci, 31:2156-2165.

[38] Mauney SA, Athanas KM, Pantazopoulos H, Shaskan N, Passeri E, Berretta S, et al. (2013). Developmental pattern of perineuronal nets in the human prefrontal cortex and their deficit in schizophrenia. Biol Psychiatry, 74:427-435.

[39] Pantazopoulos H, Woo TU, Lim MP, Lange N, Berretta S (2010). Extracellular matrix-glial abnormalities in the amygdala and entorhinal cortex of subjects diagnosed with schizophrenia. Arch Gen Psychiatry, 67:155-166.

[40] Soleman S, Filippov MA, Dityatev A, Fawcett JW (2013). Targeting the neural extracellular matrix in neurological disorders. Neuroscience, 253:194-213.

[41] Vargova L, Sykova E (2014). Astrocytes and extracellular matrix in extrasynaptic volume transmission. Philos Trans R Soc Lond B Biol Sci, 369:20130608.

[42] Ye Q, Miao QL (2013). Experience-dependent development of perineuronal nets and chondroitin sulfate proteoglycan receptors in mouse visual cortex. Matrix Biol, 32:352-363.

[43] Fawcett JW, Oohashi T, Pizzorusso T (2019). The roles of perineuronal nets and the perinodal extracellular matrix in neuronal function. Nat Rev Neurosci, 20:451-465.

[44] Itoh Y, Toriumi H, Yamada S, Hoshino H, Suzuki N (2011). Astrocytes and pericytes cooperatively maintain a capillary-like structure composed of endothelial cells on gel matrix. Brain Res, 1406:74-83. [45] Milhorat TH (1975). The third circulation revisited. J Neurosurg, 42:628-645.

[46] Han H, Li K, Yan J, Zhu K, Fu Y (2012). An in vivo study with an MRI tracer method reveals the biophysical properties of interstitial fluid in the rat brain. Sci China Life Sci, 55:782-787.

[47] Edsbagge M, Tisell M, Jacobsson L, Wikkelso C (2004). Spinal CSF absorption in healthy individuals. Am J Physiol Regul Integr Comp Physiol, 287:R1450- 
1455.

[48] Perez-Figares JM, Jimenez AJ, Rodriguez EM (2001). Subcommissural organ, cerebrospinal fluid circulation, and hydrocephalus. Microsc Res Tech, 52:591-607.

[49] Kodali M, Castro OW, Kim DK, Thomas A, Shuai B, Attaluri S, et al. (2020). Intranasally Administered Human MSC-Derived Extracellular Vesicles Pervasively Incorporate into Neurons and Microglia in both Intact and Status Epilepticus Injured Forebrain. Int J Mol Sci, 21:181.

[50] Long Q, Upadhya D, Hattiangady B, Kim DK, An SY, Shuai B, et al. (2017). Intranasal MSC-derived A1exosomes ease inflammation, and prevent abnormal neurogenesis and memory dysfunction after status epilepticus. Proc Natl Acad Sci U S A, 114:E3536E3545.

[51] Alberts BJ, A.; Lewis, J.; Raff, M.; Roberts, K.; Walter, P. 2007. Molecular Biology of the Cell. New York: Garland Science.

[52] Arish M, Husein A, Kashif M, Sandhu P, Hasnain SE, Akhter Y, et al. (2015). Orchestration of membrane receptor signaling by membrane lipids. Biochimie, 113:111-124.

[53] Bouter A, Carmeille R, Gounou C, Bouvet F, Degrelle SA, Evain-Brion D, et al. (2015). Review: AnnexinA5 and cell membrane repair. Placenta, 36 Suppl 1:S43-49.

[54] Gu C, Barry J (2011). Function and mechanism of axonal targeting of voltage-sensitive potassium channels. Prog Neurobiol, 94:115-132.

[55] Nicolson GL (2015). Cell membrane fluid-mosaic structure and cancer metastasis. Cancer Res, 75:11691176.

[56] Ide K, Secher NH (2000). Cerebral blood flow and metabolism during exercise. Prog Neurobiol, 61:397414.

[57] Mabuchi T, Lucero J, Feng A, Koziol JA, del Zoppo GJ (2005). Focal cerebral ischemia preferentially affects neurons distant from their neighboring microvessels. J Cereb Blood Flow Metab, 25:257-266.

[58] Cuddapah VA, Zhang SL, Sehgal A (2019). Regulation of the Blood-Brain Barrier by Circadian Rhythms and Sleep. Trends Neurosci, 42:500-510.

[59] Shetty AK, Mishra V, Kodali M, Hattiangady B (2014). Blood brain barrier dysfunction and delayed neurological deficits in mild traumatic brain injury induced by blast shock waves. Front Cell Neurosci, 8:232.

[60] Brasnjevic I, Steinbusch HW, Schmitz C, MartinezMartinez P, European NanoBioPharmaceutics Research I (2009). Delivery of peptide and protein drugs over the blood-brain barrier. Prog Neurobiol, 87:212-251.

[61] Banks WA. (2009). Characteristics of compounds that cross the blood-brain barrier. BMC Neurol, 9 Suppl $1:$ S3.

[62] Verheggen ICM, Van Boxtel MPJ, Verhey FRJ, Jansen JFA, Backes WH (2018). Interaction between bloodbrain barrier and glymphatic system in solute clearance. Neurosci Biobehav Rev, 90:26-33.
Deco G, Rolls ET, Albantakis L, Romo R (2013). Brain mechanisms for perceptual and reward-related decision-making. Prog Neurobiol, 103:194-213.

Edwards TN, Meinertzhagen IA (2010). The functional organisation of glia in the adult brain of Drosophila and other insects. Prog Neurobiol, 90:471497.

He C, Chen F, Li B, Hu Z (2014). Neurophysiology of $\mathrm{HCN}$ channels: from cellular functions to multiple regulations. Prog Neurobiol, 112:1-23.

Kastellakis G, Cai DJ, Mednick SC, Silva AJ, Poirazi P (2015). Synaptic clustering within dendrites: an emerging theory of memory formation. Prog Neurobiol, 126:19-35.

Zougman A, Pilch B, Podtelejnikov A, Kiehntopf M, Schnabel C, Kumar C, et al. (2008). Integrated analysis of the cerebrospinal fluid peptidome and proteome. J Proteome Res, 7:386-399.

Hobson JA, Friston KJ (2012). Waking and dreaming consciousness: neurobiological and functional considerations. Prog Neurobiol, 98:82-98.

Lo EH, Wang X, Cuzner ML (2002). Extracellular proteolysis in brain injury and inflammation: role for plasminogen activators and matrix metalloproteinases. J Neurosci Res, 69:1-9.

Melchor JP, Strickland S (2005). Tissue plasminogen activator in central nervous system physiology and pathology. Thromb Haemost, 93:655-660.

Conant K, McArthur JC, Griffin DE, Sjulson L, Wahl LM, Irani DN (1999). Cerebrospinal fluid levels of MMP-2, 7, and 9 are elevated in association with human immunodeficiency virus dementia. Ann Neurol, 46:391-398

Bonneh-Barkay D, Wiley CA (2009). Brain extracellular matrix in neurodegeneration. Brain Pathol, 19:573-585.

Holth JK, Fritschi SK, Wang C, Pedersen NP, Cirrito JR, Mahan TE, et al. (2019). The sleep-wake cycle regulates brain interstitial fluid tau in mice and CSF tau in humans. Science, 363:880-884.

Rasmussen MK, Mestre H, Nedergaard M (2018). The glymphatic pathway in neurological disorders. Lancet Neurol, 17:1016-1024.

Fultz NE, Bonmassar G, Setsompop K, Stickgold RA, Rosen BR, Polimeni JR, et al. (2019). Coupled electrophysiological, hemodynamic, and cerebrospinal fluid oscillations in human sleep. Science, 366:628-631.

Flamant S, Tamarat R (2016). Extracellular Vesicles and Vascular Injury: New Insights for Radiation Exposure. Radiat Res, 186:203-218.

Malloci M, Perdomo L, Veerasamy M, Andriantsitohaina R, Simard G, Martinez MC (2019). Extracellular Vesicles: Mechanisms in Human Health and Disease. Antioxid Redox Signal, 30:813-856.

Raposo G, Stoorvogel W (2013). Extracellular vesicles: exosomes, microvesicles, and friends. J Cell Biol, 200:373-383.

EL Andaloussi S, Mäger I, Breakefield XO, Wood MJ (2013). Extracellular vesicles: biology and emerging 
therapeutic opportunities. Nat Rev Drug Discov, 12:347-357.

[80] Gitai DLG, Dos Santos YDR, Upadhya R, Kodali M, Madhu LN, Shetty AK (2019). Extracellular Vesicles in the Forebrain Display Reduced miR-346 and miR331-3p in a Rat Model of Chronic Temporal Lobe Epilepsy. Mol Neurobiol.

[81] Goetzl EJ, Boxer A, Schwartz JB, Abner EL, Petersen $\mathrm{RC}$, Miller BL, et al. (2015). Altered lysosomal proteins in neural-derived plasma exosomes in preclinical Alzheimer disease. Neurology, 85:40-47.

[82] Goetzl EJ, Kapogiannis D, Schwartz JB, Lobach IV, Goetzl L, Abner EL, et al. (2016). Decreased synaptic proteins in neuronal exosomes of frontotemporal dementia and Alzheimer's disease. FASEB J, 30:41414148.

[83] Kanninen KM, Bister N, Koistinaho J, Malm T (2016). Exosomes as new diagnostic tools in CNS diseases. Biochim Biophys Acta, 1862:403-410.

[84] Madhu LN, Attaluri S, Kodali M, Shuai B, Upadhya R, Gitai D, et al. (2019). Neuroinflammation in Gulf War Illness is linked with HMGB1 and complement activation, which can be discerned from brain-derived extracellular vesicles in the blood. Brain Behav Immun, 81:430-443.

[85] Upadhya D, Shetty AK (2019). Promise of extracellular vesicles for diagnosis and treatment of epilepsy. Epilepsy Behav:106499.

[86] Johanson C, Stopa E, McMillan P, Roth D, Funk J, Krinke G (2011). The distributional nexus of choroid plexus to cerebrospinal fluid, ependyma and brain: toxicologic/pathologic phenomena, periventricular destabilization, and lesion spread. Toxicol Pathol, 39:186-212.

[87] Pollock H, Hutchings M, Weller RO, Zhang ET (1997). Perivascular spaces in the basal ganglia of the human brain: their relationship to lacunes. J Anat, 191 ( Pt 3):337-346.

[88] Han H, Shi C, Fu Y, Zuo L, Lee K, He Q, et al. (2014). A novel MRI tracer-based method for measuring water diffusion in the extracellular space of the rat brain. IEEE J Biomed Health Inform, 18:978-983.

[89] Bradbury MW, Cserr HF, Westrop RJ (1981). Drainage of cerebral interstitial fluid into deep cervical lymph of the rabbit. Am J Physiol, 240:F329-336.

[90] Carare RO, Bernardes-Silva M, Newman TA, Page AM, Nicoll JA, Perry VH, et al. (2008). Solutes, but not cells, drain from the brain parenchyma along basement membranes of capillaries and arteries: significance for cerebral amyloid angiopathy and neuroimmunology. Neuropathol Appl Neurobiol, 34:131-144.

[91] Lonser RR, Sarntinoranont M, Morrison PF, Oldfield EH (2015). Convection-enhanced delivery to the central nervous system. J Neurosurg, 122:697-706.

[92] Louveau A, Smirnov I, Keyes TJ, Eccles JD, Rouhani SJ, Peske JD, et al. (2015). Structural and functional features of central nervous system lymphatic vessels. Nature, 523:337-341.

[93] Shi C, Lei Y, Han H, Zuo L, Yan J, He Q, et al. (2015).
Transportation in the Interstitial Space of the Brain Can Be Regulated by Neuronal Excitation. Sci Rep, 5:17673.

[94] Kiviniemi V, Wang X, Korhonen V, Keinanen T, Tuovinen T, Autio J, et al. (2016). Ultra-fast magnetic resonance encephalography of physiological brain activity - Glymphatic pulsation mechanisms? J Cereb Blood Flow Metab, 36:1033-1045.

[95] Nakada T, Kwee IL (2019). Fluid Dynamics Inside the Brain Barrier: Current Concept of Interstitial Flow, Glymphatic Flow, and Cerebrospinal Fluid Circulation in the Brain. Neuroscientist, 25:155-166.

[96] Teng Z, Wang A, Wang P, Wang R, Wang W, Han H (2018). The effect of aquaporin-4 knockout on interstitial fluid flow and the structure of the extracellular space in the deep brain. Aging Dis, 9:808-816.

[97] Weller RO, Hawkes CA, Kalaria RN, Werring DJ, Carare RO (2015). White matter changes in dementia: role of impaired drainage of interstitial fluid. Brain Pathol, 25:63-78.

[98] Lv D, Li J, Li H, Fu Y, Wang W (2017). Imaging and Quantitative Analysis of the Interstitial Space in the Caudate Nucleus in a Rotenone-Induced Rat Model of Parkinson's Disease Using Tracer-based MRI. Aging Dis, 8:1-6.

[99] Zhang Y, Sun L, Liu E, Wang A, Yan J (2019). The olfactory stimulation slows down the substance clearance in the extracellular space of the hippocampus in rat brain. Biochem Biophys Res Commun, 515:429-435.

[100] Kingsmore KM, Vaccari A, Abler D, Cui SX, Epstein FH, Rockne RC. et al. (2018). MRI analysis to map interstitial flow in the brain tumor microenvironment. APL Bioeng, 2(3). pii: 031905.

[101] Howe MD, Atadja LA, Furr JW, Maniskas ME, Zhu L, McCullough LD, Urayama A. Fibronectin induces the perivascular deposition of cerebrospinal fluid-derived amyloid- $\beta$ in aging and after stroke. Neurobiol Aging, 2018, 72:1-13.

[102] Birlik B, Canda S, Ozer E (2006). Tumour vascularity is of prognostic significance in adult, but not paediatric astrocytomas. Neuropathol Appl Neurobiol, 32:532538.

[103] Vargova L, Homola A, Zamecnik J, Tichy M, Benes V, Sykova E (2003). Diffusion parameters of the extracellular space in human gliomas. Glia, 42:77-88.

[104] Zamecnik J (2005). The extracellular space and matrix of gliomas. Acta Neuropathol, 110:435-442.

[105] Guan XW, W.; Wan, A.; Teng, Z.; Han, H. (2018). Brain Interstitial Fluid Drainage Alterations in Glioma-Bearing Rats. Aging and disease, 9:228-234.

[106] Steiner E, Enzmann GU, Lin S, Ghavampour S, Hannocks MJ, Zuber B, et al. (2012). Loss of astrocyte polarization upon transient focal brain ischemia as a possible mechanism to counteract early edema formation. Glia, 60:1646-1659.

[107] Hrabetova S, Nicholson C. 2007. Biophysical Properties of Brain Extracellular Space Explored with Ion-Selective Microelectrodes, Integrative Optical 
Imaging and Related Techniques. In Electrochemical Methods for Neuroscience. A.C. Michael, and L.M. Borland, editors. Boca Raton (FL).

[108] Sykova E, Svoboda J, Polak J, Chvatal A (1994). Extracellular volume fraction and diffusion characteristics during progressive ischemia and terminal anoxia in the spinal cord of the rat. J Cereb Blood Flow Metab, 14:301-311.

[109] Vorisek I, Sykova E (1997). Ischemia-induced changes in the extracellular space diffusion parameters, $\mathrm{K}+$, and $\mathrm{pH}$ in the developing rat cortex and corpus callosum. J Cereb Blood Flow Metab, 17:191-203.

[110] Liu E, Sun L, Zhang Y, Wang A, Yan J (2020). Aquaporin4 Knockout Aggravates Early Brain Injury Following Subarachnoid Hemorrhage Through Impairment of the Glymphatic System in Rat Brain. Acta Neurochir Suppl, 127:59-64.

[111] Burns A, Iliffe S (2009). Alzheimer's disease. BMJ, 338:b158.

[112] Mudher A, Lovestone S (2002). Alzheimer's diseasedo tauists and baptists finally shake hands? Trends Neurosci, 25:22-26.

[113] Nistor M, Don M, Parekh M, Sarsoza F, Goodus M, Lopez GE, et al. (2007). Alpha- and beta-secretase activity as a function of age and beta-amyloid in Down syndrome and normal brain. Neurobiol Aging, 28:1493-1506.

[114] Arbel-Ornath M, Hudry E, Eikermann-Haerter K, Hou S, Gregory JL, Zhao L, et al. (2013). Interstitial fluid drainage is impaired in ischemic stroke and Alzheimer's disease mouse models. Acta Neuropathol, 126:353-364.

[115] Greenberg SM, Bacskai BJ, Hernandez-Guillamon M, Pruzin J, Sperling R, van Veluw SJ (2019). Cerebral amyloid angiopathy and Alzheimer disease - one peptide, two pathways. Nat Rev Neurol.

[116] Marti M, Sarubbo S, Latini F, Cavallo M, Eleopra R, Biguzzi S, et al. (2010). Brain interstitial nociceptin/orphanin FQ levels are elevated in Parkinson's disease. Mov Disord, 25:1723-1732.

[117] Louveau A, Da Mesquita S, Kipnis J (2016). Lymphatics in Neurological Disorders: A NeuroLympho-Vascular Component of Multiple Sclerosis and Alzheimer's Disease? Neuron, 91:957-973.

[118] Nikolenko VN, Oganesyan MV, Vovkogon AD, Nikitina AT, Sozonova EA, Kudryashova VA et al. (2019). Current Understanding of Central Nervous System Drainage Systems: Implications in the Context of Neurodegenerative Diseases. Curr Neuropharmacol.

[119] Rogawski MA (2009). Convection-enhanced delivery in the treatment of epilepsy. Neurotherapeutics, 6:344351.

[120] Cheng Y, Liu X, Ma X, Garcia R, Belfield K, Haorah $\mathrm{J}$ (2019). Alcohol promotes waste clearance in the CNS via brain vascular reactivity. Free Radic Biol Med, 143:115-126.

[121] Pangalos MN, Schechter LE, Hurko O (2007). Drug development for CNS disorders: strategies for balancing risk and reducing attrition. Nat Rev Drug Discov, 6:521-532.
[122] Wolak DJ, Thorne RG (2013). Diffusion of macromolecules in the brain: implications for drug delivery. Mol Pharm, 10:1492-1504.

[123] Gribkoff VK, Kaczmarek LK (2017). The need for new approaches in CNS drug discovery: Why drugs have failed, and what can be done to improve outcomes. Neuropharmacology, 120:11-19.

[124] Modi G, Pillay V, Choonara YE, Ndesendo VM, du Toit LC, Naidoo D (2009). Nanotechnological applications for the treatment of neurodegenerative disorders. Prog Neurobiol, 88:272-285.

[125] Anderson RC, Kennedy B, Yanes CL, Garvin J, Needle M, Canoll P, et al. (2013). Convectionenhanced delivery of topotecan into diffuse intrinsic brainstem tumors in children. J Neurosurg Pediatr, 11:289-295.

[126] Barua NU, Gill SS, Love S (2014). Convectionenhanced drug delivery to the brain: therapeutic potential and neuropathological considerations. Brain Pathol, 24:117-127.

[127] Barua NU, Woolley M, Bienemann AS, Johnson DE, Lewis O, Wyatt MJ, et al. (2013). Intermittent convection-enhanced delivery to the brain through a novel transcutaneous bone-anchored port. J Neurosci Methods, 214:223-232.

[128] Mehta AM, Sonabend AM, Bruce JN (2017). Convection-Enhanced Delivery. Neurotherapeutics, 14:358-371.

[129] Bruce JN, Fine RL, Canoll P, Yun J, Kennedy BC, Rosenfeld SS, et al. (2011). Regression of recurrent malignant gliomas with convection-enhanced delivery of topotecan. Neurosurgery, 69:1272-1279; discussion 1279-1280.

[130] Lidar Z, Mardor Y, Jonas T, Pfeffer R, Faibel M, Nass $\mathrm{D}$, et al. (2004). Convection-enhanced delivery of paclitaxel for the treatment of recurrent malignant glioma: a phase I/II clinical study. J Neurosurg, 100:472-479.

[131] Slevin JT, Gash DM, Smith CD, Gerhardt GA, Kryscio $\mathrm{R}$, Chebrolu H, et al. (2006). Unilateral intraputaminal glial cell line-derived neurotrophic factor in patients with Parkinson disease: response to 1 year each of treatment and withdrawal. Neurosurg Focus, 20:E1.

[132] Barua NU, Bienemann AS, Hesketh S, Wyatt MJ, Castrique E, Love S, et al. (2012). Intrastriatal convection-enhanced delivery results in widespread perivascular distribution in a pre-clinical model. Fluids Barriers CNS, 9:2.

[133] Barua NU, Miners JS, Bienemann AS, Wyatt MJ, Welser K, Tabor AB, et al. (2012). Convectionenhanced delivery of neprilysin: a novel amyloid-betadegrading therapeutic strategy. J Alzheimers Dis, 32:43-56.

[134] Sampson JH, Raghavan R, Brady ML, Provenzale JM, Herndon JE, 2nd, Croteau D, et al. (2007). Clinical utility of a patient-specific algorithm for simulating intracerebral drug infusions. Neuro Oncol, 9:343-353.

[135] Han H, Xia Z, Chen H, Hou C, Li W (2011). Simple diffusion delivery via brain interstitial route for the treatment of cerebral ischemia. Sci China Life Sci, 
54:235-239.

[136] Xu F, Han H, Zhang H, Pi J, Fu Y (2011). Quantification of Gd-DTPA concentration in neuroimaging using T(1)3D MP-RAGE sequence at 3.0 T. Magn Reson Imaging, 29:827-834. 